\title{
The clinical value of the delta finger to palm distance in systemic sclerosis
}

\author{
A. Javinani ${ }^{1}$, S. Mostafaei ${ }^{2}$, F. Gharibdoost ${ }^{1}$, A.R. Jamshidi', \\ R. Atef Yekta ${ }^{3}$, T. Kiani Fard', Z. Tamartash'1, F. Gorgani' ${ }^{1}$, M. Mirbeyk', \\ M. Mahmoudi', K. Sadeghi ${ }^{4}$, H. Kavosi ${ }^{1}$ \\ ${ }^{1}$ Rheumatology Research Center, Tehran University of Medical Sciences, Tehran, Iran; \\ ${ }^{2}$ Medical Biology Research Center, Health Technology Institute, Kermanshah University of Medical Sciences, \\ Kermanshah, Iran; \\ ${ }^{3}$ Anesthesiology Department, Shariati Hospital, Tehran University of Medical Sciences, Tehran, Iran; \\ ${ }^{4}$ Department of Clinical Pharmacy, Tehran University of Medical Sciences, Tehran, Iran
}

\section{SUMMARY}

Systemic sclerosis (SSc) is a collagen-vascular disorder characterized by fibrosis and vasculopathy. Delta finger to palm distance (delta FTP) is an index measuring the distance between the tip of the third finger to the distal palmar crease in the flexed and extended position. The present study aimed to evaluate the clinical value of delta FTP and to assess the correlation of delta FTP with modified Rodnan skin score (mRSS) and forced vital capacity (FVC) over the 12-month follow-up.

This prospective longitudinal study began with 50 participants who were followed for twelve months. Lowess smoothing and linear regression were applied to detect and assess the relationship between delta FTP and mRSS. p-values were adjusted by the Benjamini-Hochberg method (BHM) as a control for false discovery rate. Delta FTP was lower among patients with higher disease duration (p-value ${ }^{\text {adj: }}$ 0.008), diffuse cutaneous SSc (p-value adj: 0.006), digital ulcers ( $p$-value ${ }^{\text {adj }}$ : 0.003), telangiectasia (p-value ${ }^{\text {adj: }}$ 0.006) and dysphagia (p-value ${ }^{\text {adj: }}$ 0.036). The mRSS has a significant negative linear effect on the delta FTP at the baseline and the end of the follow-up ( $r$ : -0.31 and -0.40 , respectively). Moreover, changes of mRSS and delta FTP showed a negative linear association over time $(r:-0.22)$. These linear effects remained significant after regrouping the patients based on their SSc subtype. Delta FTP and FVC were not correlated either at the baseline or at the end.

It seems that the delta FTP can be a valuable clinical index, supported by its correlated changes with mRSS and other SSc clinical manifestations over the one-year follow-up.

Key words: Scleroderma, systemic, outcome assessment, tendons.

Reumatismo, 2020; 72 (1): 44-51

\section{INTRODUCTION}

ystemic sclerosis $(\mathrm{SSc})$ is a member of the collagen vascular disorder family that is chiefly distinguished from its other counterparts by the prominent fibrosis of multiple organs, which is the hallmark of this disease (1). Skin stiffness and tendon fibrosis are manifestations that are experienced early in the course of the disease, noticed more prominently in the extremities. The cutaneous and tendon fibrosis lead to a decreased range of motion in the hands, mainly when they occur alongside with arthropathies, and may eventually result in flexion contracture $(2,3)$.

In regular examinations of SSc patients, specific indices are used to assess the extent of fibrosis and dysfunction in different organs, which are primarily applied to evaluate treatment efficacy. These indices should be easily-performed, cost-effective and feasible. The most popular index used in the clinic and research settings is the modified Rodnan skin score (mRSS) (46). This index is a 0-3 subjective scoring system of 17 areas, used to evaluate the skin stiffness. This index has become increasingly popular due to its feasibility and its association with other internal organs manifestations, including pulmonary and cardiac involvements $(7,8)$. Another index that was firstly presented in Medsger's SSc severity score (MSSS) is the finger to palm 
distance (FTP), defined by the distance between the tip of the third finger to the distal palmar crease in the flexed position (9). FTP can be a measure of the hands' range of motion and indirectly influenced by the skin stiffness, tendon fibrosis, and arthropathies. Later, the delta FTP was introduced to measure the distance between the tip of the third finger to the distal palmar crease in the flexed and extended position and to record the difference between these two values (10).

The purpose of this study was to investigate the association of delta FTP with demographic features, cutaneous, gastrointestinal, pulmonary and cardiac manifestations. To better assess the probable utility of delta FTP in patients follow-up, we have evaluated the delta FTP changes over one year. Ultimately, our secondary aim was to investigate the association of delta FTP changes with mRSS and forced vital capacity (FVC) changes over the follow-up period.

\section{PATIENTS AND METHODS}

\section{Study design}

In this study, fifty patients were enrolled from the SSc registry of the Rheumatology Research Center (RRC) of Shariati hospital, Tehran University of Medical Sciences (TUMS), by a simple random sampling approach. To more precisely evaluate the correlation between delta FTP and other clinical indices; patients with active synovitis, digital gangrenes and auto-amputations were excluded. The participants fulfilled the SSc criteria according to the 2013 classification criteria for systemic sclerosis: an American College of Rheumatology/European League Against Rheumatism (ACR/ EULAR) collaborative initiative (11).

Forty-three patients were also prospectively followed up for 12 months to investigate the correlation of delta FTP changes with mRSS and FVC alteration. The delta FTP, mRSS and FVC were re-measured at the end of the follow-up period. To adjust for the confounding effect of SSc subtype and disease duration on this relationship, all of the analyses were adjusted based on these two confounders. This study was started in October 2015 and ended in May 2017.

\section{Demographic, clinical and laboratory data}

Demographic features were collected at the beginning of the study. SSc subtypes were reported according to the 2013 ACR/ EULAR classification (11). Disease duration was calculated from the Raynaud's phenomenon to the time of the study commencement.

The gastrointestinal and cutaneous symptoms were recorded according to the history and the physical examination. Delta FTP was defined as the difference between the tip of the third finger to the distal palmar crease in the extended and flexed position (10). The mRSS was calculated by one rheumatologist (HK) with an acceptable reliability coefficient over time ( $\mathrm{ICC}=0.88$ ). Cardiac involvement was assessed by transthoracic echocardiogram, and the estimated pulmonary arterial pressure (PAP) and ejection fraction $(\mathrm{EF})$ were reported. Interstitial lung disease (ILD) was defined according to the pulmonary imaging studies, including either high resolution computed tomography or chest $\mathrm{x}$-ray.

\section{Statistical analysis}

In the present study, the variables were checked for normal distribution by the Shapiro-Wilk test. The delta FTP variable had a non-parametric distribution. Accordingly, to investigate the correlation of delta FTP with other demographic and clinical indices, the Spearman's Rank-Order correlation, Mann-Whitney test, and chi-square $\left(\chi^{2}\right)$ test were used.

To evaluate the association of the changes in delta FTP with mRSS and FVC, the scatter plot (Lowess smoothing) was used to assess the shape of their associations. Lowess smoothing recognized that the delta FTP has a linear association with mRSS and FVC. Subsequently, the linear regression was applied to analyze their association. To adjust for the confounding effect of SSc subtype and disease duration, they were considered as independent variables in the multiple linear regression 
Table I - Demographic and clinical disease characteristics of the SSc patients.

\begin{tabular}{|l|c|c|}
\hline \multirow{2}{*}{ Variable } & Subgroup & $\begin{array}{c}\text { Patients (n: 50) } \\
\text { Mean } \pm \text { SD } \\
\mathbf{N}(\%) \\
\text { Median (IQR }\end{array}$ \\
\hline Age (year) & - & $40.1 \pm 10.3$ \\
\hline \multirow{2}{*}{ Sex } & Female & Reference \\
\hline Disease duration (year) & Male & $10(20.0 \%)$ \\
\hline \multirow{2}{*}{ Subtype } & - & $7.2 \pm 4.3$ \\
\hline Delta FTP $(\mathrm{cm})$ & Limited & $23(46.0 \%)$ \\
\cline { 2 - 3 } & Diffuse & $27(54.0 \%)$ \\
\hline
\end{tabular}

FTP, finger to palm ratio; SD, standard deviation; IQR, interquartile range.

model. All of the statistical tests were done using STATA 11.0 (STATA Corp, College Station, TX). p-value $<0.05$ were considered as statistically significant. p-values were adjusted by the Benjamini-Hochberg method (BHM) as a control for false discovery rate (12).

\section{Compliance with ethical standards}

All procedures performed in studies involving human participants were in accordance with the ethical standards of the RRC and TUMS research committee. In addition, informed consent was obtained from all individual participants included in the study.

\section{RESULTS}

\section{The association of delta FTP} with demographic and clinical variables

The demographic and clinical data of fifty SSc participants are shown in Table I. The association of delta FTP with these variables are shown in Table II. Patients with limited cutaneous SSc (lcSSc) had higher

Table II - The association between delta FTP and clinical manifestations/laboratory indices.

\begin{tabular}{|c|c|c|c|}
\hline Variable & Subgroup & $\begin{array}{c}\text { Median }\left(1 Q R_{25-75}\right) \\
\text { Correlation coefficient }\end{array}$ & $p$-value adj \\
\hline Age (year) ${ }^{*}$ & - & 0.023 & 0.808 \\
\hline \multirow{2}{*}{ Sex } & Female & $6.0(4.5-7.0)$ & \multirow{2}{*}{0.378} \\
\hline & Male & $6.5(5.0-7.0)$ & \\
\hline Disease duration (year) $^{\star}$ & - & -0.288 & 0.008 \\
\hline \multirow{2}{*}{ Subtype } & Limited & $7.0(5.38-7.5)$ & \multirow{2}{*}{0.006} \\
\hline & Diffuse & $5.0(4.5-7.0)$ & \\
\hline $\operatorname{BMl}\left(\mathrm{kg} / \mathrm{m}^{2}\right)^{\star}$ & - & 0.238 & 0.033 \\
\hline \multirow{2}{*}{ GERD } & Positive & $6.0(4.5-7.0)$ & \multirow{2}{*}{0.325} \\
\hline & Negative & $6.0(5.0-7.13)$ & \\
\hline \multirow{2}{*}{ Dysphagia } & Positive & $5.5(4.0-6.8)$ & \multirow{2}{*}{0.036} \\
\hline & Negative & $6.5(5.0-7.0)$ & \\
\hline \multirow{2}{*}{ Telangiectasia } & Positive & $5.0(4.0-6.75)$ & \multirow{2}{*}{0.006} \\
\hline & Negative & $6.75(5.0-7.5)$ & \\
\hline \multirow{2}{*}{ Arthralgia } & Positive & $6.0(4.5-7.0)$ & \multirow{2}{*}{0.080} \\
\hline & Negative & $6.5(5.0-7.5)$ & \\
\hline \multirow{2}{*}{ Digital ulcer } & Positive & $5.0(3.6-6.5)$ & \multirow{2}{*}{0.003} \\
\hline & Negative & $6.5(5.0-7.0)$ & \\
\hline \multirow[t]{2}{*}{ ILD } & Positive & $5.0(1.5-7.0)$ & \multirow{2}{*}{0.325} \\
\hline & Negative & $6.75(3.13-7.5)$ & \\
\hline $\operatorname{ePAP}(\mathrm{mmHg})^{\star}$ & - & -0.105 & 0.378 \\
\hline $\mathrm{EF}(\%)^{*}$ & - & 0.058 & 0.597 \\
\hline
\end{tabular}

IQR, interquartile range; BMI, body mass index; GERD, gastro-esophageal reflux disease; ILD, interstitial lung disease; ePAP, estimated pulmonary arterial pressure; EF, ejection fraction. P-values were adjusted by Benjamini-Hochberg method; * the correlation coefficient is reported for these variables. The rest of the variables are reported by median (IQR25-75). 
delta FTP compared to patients with diffuse cutaneous SSc (dcSSc) (p-value: 0.006 ). Disease duration had a negative association with delta FTP with a correlation coefficient of -0.288 and p-value of 0.008 . The delta FTP was associated with dysphagia and telangiectasia with the $\mathrm{p}$-value of 0.036 and 0.006 , respectively. Moreover, the delta FTP was significantly higher in patients with digital ulcers (5.0 (3.6-6.5) $\mathrm{cm})$ compared to cases without ulcers (6.5 (5.0-7.0) cm, p-value: 0.003).

\section{The association of delta FTP with $\mathrm{mRSS}$ and $\mathrm{FVC}$}

By using scatter plot (Lowess smoother), the associations of delta FTP with mRSS and FVC are shown in Figure $1 \mathrm{~A}$ and B, respectively. According to the standardized linear regression, the mRSS had a significant negative linear effect on the delta FTP (p-value $<0.001$, B: -0.55 and $r$ : -0.31 ). The association of the delta FTP and mRSS was in a linear framework with the following formula: [delta FTP $=8.036-0.55 \times \mathrm{mRSS}$ ]. Whereas, the delta FTP was not statistically associated with FVC (p-value: 0.550).

\section{Prospective analysis of the delta FTP association with mRSS and FVC}

As described previously, forty-three SSc cases were followed for 12-month and the delta FTP, mRSS and FVC were re-measured at the end of the 12-month period (Figure $1 C$ and D). At the end of the follow-up, mRSS had a significant negative linear effect on the delta FTP (p-value $<0.001, \mathrm{~B}$ : -0.630 and $r:-0.40)$. The association of the

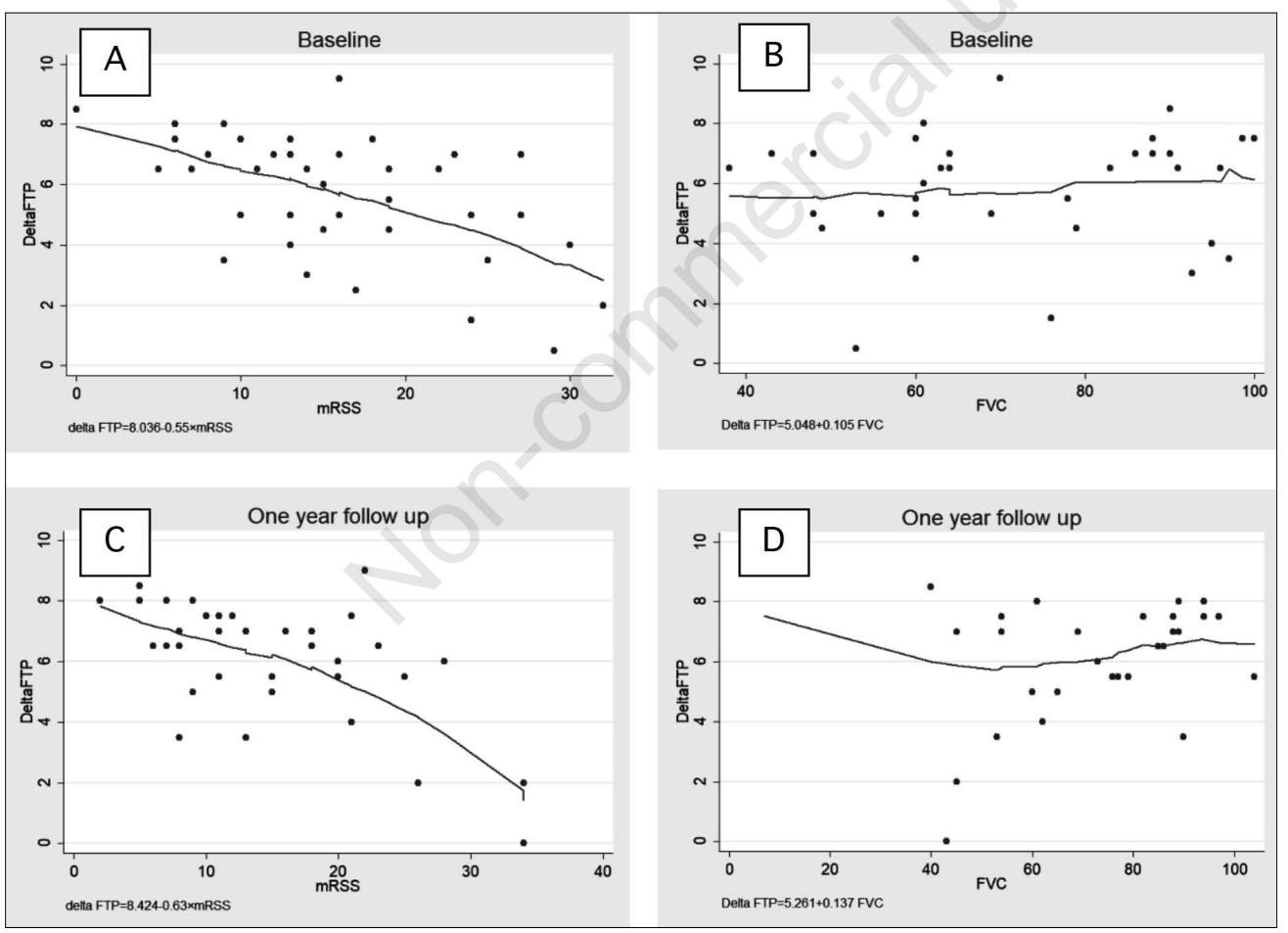

Figure 1 - The associations of delta finger to palm distance (delta FTP) with modified Rodnan skin score (mRSS) and forced vital capacity (FVC) at the baseline and the follow up. A: Linear association between delta FTP and mRSS by Lowess smoother (suppress weighted regression) and bandwidth $=0.8$ at baseline. B: Linear association between delta FTP and FVC by Lowess smoother (suppress weighted regression) and bandwidth $=0.8$ at baseline. C: Linear association between delta FTP and mRSS by Lowess smoother (suppress weighted regression) and bandwidth $=0.8$ at the end of one year follow up. D: Linear association between delta FTP and FVC by Lowess smoother (suppress weighted regression) and bandwidth $=0.8$ at the end of one year follow up. 


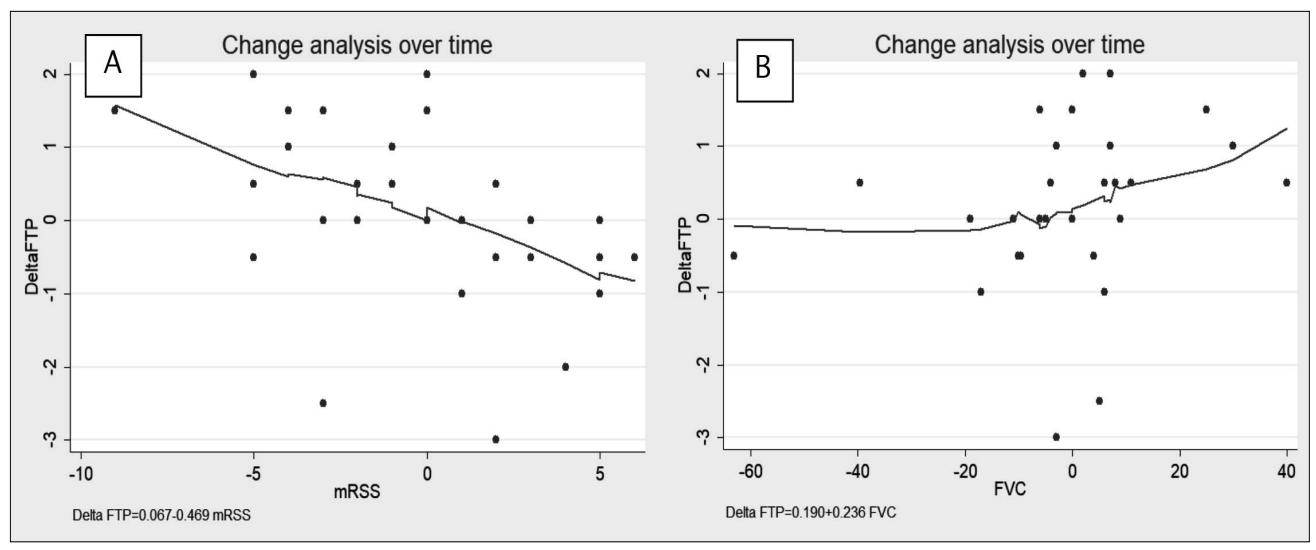

Figure 2 - The change analyses of delta finger to palm distance (delta FTP) with modified Rodnan skin score (mRSS) and forced vital capacity (FVC). A: Change analysis for detecting the linear association between delta FTP and mRSS by Lowess smoother (suppress weighted regression) and bandwidth $=0.8$ over one year follow up. B: Change analysis for detecting the linear association between delta FTP and FVC by Lowess smoother (suppress weighted regression) and bandwidth $=0.8$ over one year follow up.

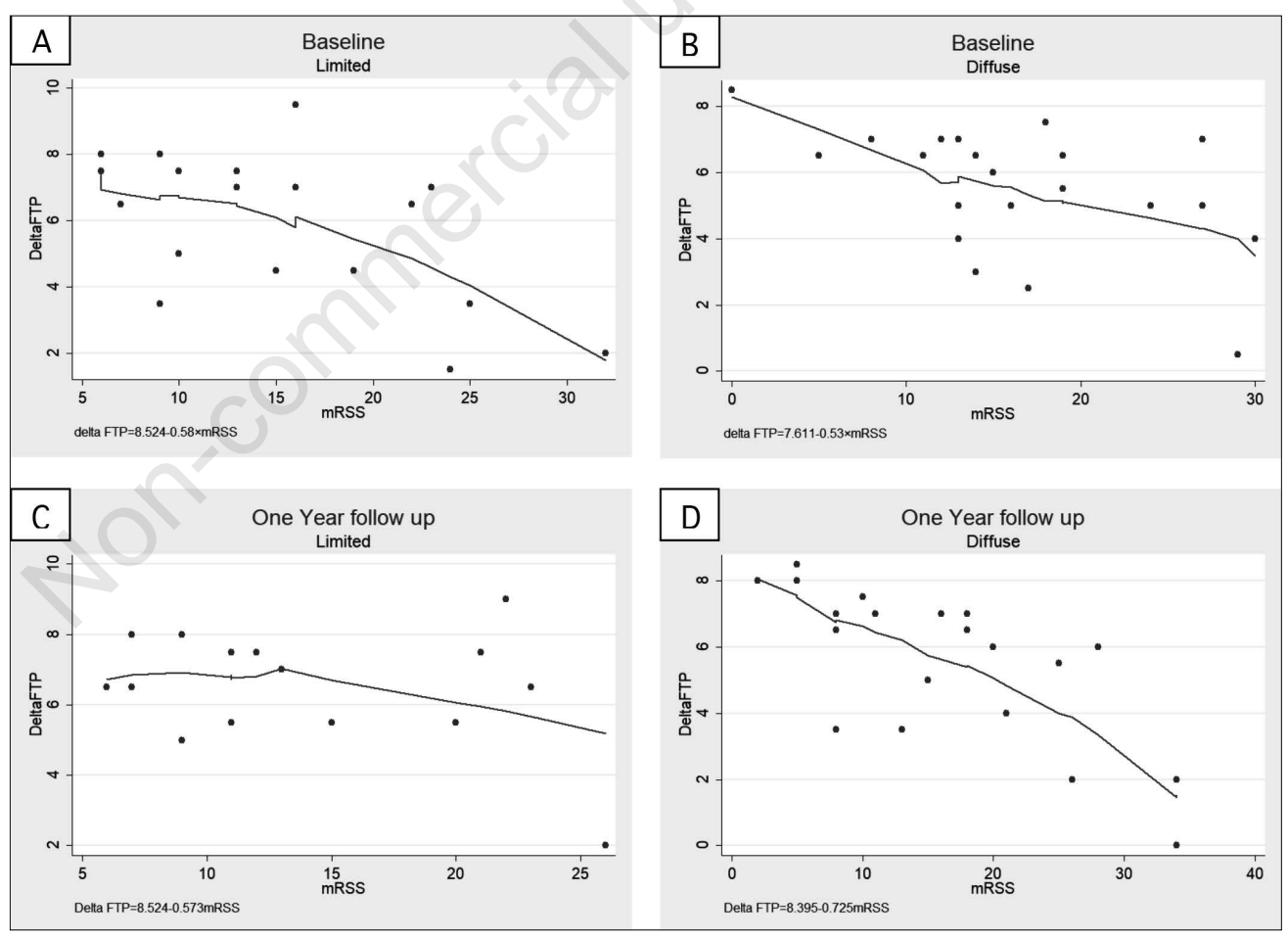

Figure 3 - The associations of delta finger to palm distance (delta FTP) with modified Rodnan skin score (mRSS) at the baseline and the follow up based on SSc subtypes. A: Linear association between delta FTP and mRSS by Lowess smoother (suppress weighted regression) and bandwidth $=0.8$ in the limited cases. B: Linear association between delta FTP and mRSS by Lowess smoother (suppress weighted regression) and bandwidth $=0.8$ in the diffuse cases. C: Linear association between delta FTP and mRSS by Lowess smoother (suppress weighted regression) and bandwidth $=0.8$ in the limited cases. D: Linear association between delta FTP and mRSS by Lowess smoother (suppress weighted regression) and bandwidth $=0.8$ in the diffuse cases. 
delta FTP and mRSS was in a linear framework with the following formula: [delta FTP $=8.424-0.63 \times \mathrm{mRSS}]$. However, the delta FTP was not statistically associated with FVC (p-value: 0.470). Furthermore, changes of mRSS and delta FTP were correlated and showed a negative linear association (p-value: $0.003, \mathrm{~B}:-0.469$ and $r$ : -0.22 ) over the one-year follow up (Figure 2A). Meanwhile, the changes of delta FTP and FVC were not correlated over this period (p-value: 0.218 ).

\section{The association of delta FTP with $\mathrm{mRSS}$ in different SSc subtypes} The association of delta FTP with mRSS among lcSSc and dcSSc patients is shown in Figure 3. Among lcSSc patients, the mRSS had a significant linear association with delta FTP in the beginning and end of the study (p-value: $<0.001$ and $<0.001$, respectively). Moreover, the changes in these two variables were also correlated during the 12-month period (p-value: 0.002). Similarly, dcSSc cases had a significant association between delta FTP and mRSS in both the baseline and the end of the study (p-value: $<0.001$ and $<0.001$, respectively). Additionally, there was a trend toward correlated changes of these two variables during the follow-up, although it was not statistically significant (p-value: 0.069).

\section{DISCUSSION}

The current study was primarily designed to investigate the clinical value of delta FTP among SSc patients. We have reported a significant linear association of delta FTP with mRSS. This result was replicated in the baseline and after a 12-month follow up among Iranian SSc patients. Moreover, this linear association remained significant after regrouping the patients based on their SSc subtype. Additionally, the changes in these two parameters were also correlated significantly over the time of the follow-up. Therefore, it appears that their association is independent of the disease subtype. Similar to our study, Khanna et al. have investigated the association of tendon friction rub (TFR) changes over 12-month with mRSS changes among early dcSSc patients (13). They showed that change in TFR and baseline mRSS predicted the change in mRSS over twelve months. Similarly, the EUSTAR analysis demonstrated that patients without TFR at the baseline are more likely to regress in their mRSS in the next 12 months (14). In line with our findings, Tas and colleagues have also reported the significant association between delta FTP and mRSS (15). It was also reported previously among American patients with early dcSSc that the TFR is associated with higher mRSS (16). Taken together, these observations provide strong evidence that the delta FTP and TFR are closely associated with mRSS.

The present study highlights the significant association of delta FTP and digital pitting ulcers. Similarly, the EUSTAR registry has shown the significant association of TFR with joint contracture and digital ulcers (17). Moreover, the multivariate analysis reinforced the correlation of hand and finger mRSS with digital ulcer development in the Thai SSc registry (18). The association of the digital ulcers and delta FTP can be justified by the probable association of hand movement and blood circulation in the fingers. Several studies reported the association of FTP and (99m) Tc-DTPA imaging in detecting microvascular involvement in patients with Raynaud's phenomenon $(19,20)$. They compared the microvascular disturbances in two different subsets of patients with primary and secondary Raynaud's phenomenon. Vascular impairment was predominantly detected in individuals with secondary Raynaud's phenomenon, which was also correlated with FTP. These findings can be further investigated in the future by the longitudinal studies showing the probable effective role of hand exercises on micro-vascular impairments and the formation of new digital ulcers.

In our study, delta FTP was positively associated with BMI, which means that patients with higher BMI have less flexion contracture. Doré and colleagues also reported that early dcSSc patients with TFR had lower BMI (16). Among the healthy population, 
the correlation of the elasticity and diameters of Achilles tendon with demographic features had been previously studied. It has been shown that BMI does not have any influence on Achilles tendon elasticity (21). So it appears that the association of tendon fibrosis and BMI among SSc patients is related to disease activity per se. It was known previously that higher SSc disease activity is associated with cachexia and lower BMI in addition to tendon fibrosis $(22,23)$. Consequently, the association of delta FTP with BMI can be explained by the indirect effect of disease activity on either of the two variables.

To further investigate the clinical value of delta FTP, its association with FVC was also analyzed. In our study, delta FTP and FVC were associated neither in the baseline nor at the end of the follow-up. Their changes throughout the 12 months were also not correlated. In contrast to our study, Tas et al. reported a significant correlation between FTP and lung involvement among their SSc patients (15). Similarly, TFR was also associated with pulmonary fibrosis and restrictive lung disease in the American SSc population (16). However, they demonstrated that TFR found at the first examination of early dcSSc patients could not predict pulmonary involvement in cases with spared lung disease at their presentation (16). Furthermore, the association of TFR and joint contracture with pulmonary fibrosis was also reported among the EUSTAR SSc registry (17). Lack of the association between delta FTP and FVC in our study group can be explained by the absence of the association between pulmonary involvement and mRSS among the Iranian SSc population (1). It seems that the association of TFR with pulmonary involvement can be explained by the correlation of skin stiffness with both variables. This finding may suggest that tendon involvement is primarily associated with mRSS rather than lung involvement.

Although this study has reached its primary aims, certain limitations need to be declared. In the present study, the number of patients followed was not adequate to reach a consensus. In addition, SSc is an illness with a slow progression rate without overt flare-ups. Consequently, it is recommended that this study be replicated elsewhere to cross-validate our findings in different populations with larger sample sizes and longer follow-up times to better evaluate the clinical value of delta FTP.

In conclusion, it seems that the delta FTP is a forgotten clinical marker that should be entered at the bedside of the SSc patients. This clinical index is accessible and feasible without either dependence on physicians' expertise or inter- and intra-personal variation in its measurement. The present study highlights that delta FTP is closely associated with mRSS, supported by their correlated changes over the 12-month follow-up. This association is independent of the disease duration and SSc subtype. Moreover, delta FTP is also associated with other clinical variables including digital ulcers, which can be a leading point toward further clinical studies.

\section{Conflict of interest}

The authors declare that there is not any conflict of interest.

\section{REFERENCES}

1. Allanore Y, Gharibdoost F, Jamshidi AR, et al. Comparison of the clinical phenotype of systemic sclerosis patients in Iran and France in two university centers. J Scleroderma Related Disord. 2018; 2397198318809224.

2. Avouac J, Guerini H, Wipff J, et al. Radiological hand involvement in systemic sclerosis. Ann Rheum Dis. 2006; 65: 1088-92.

3. Varjú C, Péntek M, Lóránd V, et al. Musculoskeletal Involvement in Systemic Sclerosis: An Unexplored Aspect of the Disease. J Scleroderma Related Disord. 2017; 2: 19-32.

4. Tyndall AJ, Bannert B, Vonk M, et al. Causes and risk factors for death in systemic sclerosis: a study from the EULAR Scleroderma Trials and Research (EUSTAR) database. Ann Rheum Dis. 2010; 69: 1809-15.

5. Javinani A, Kavosi H. Systemic sclerosisrelated myopathy. Rheumatol Res. 2017; 2: 85-9.

6. Furst DE, Clements PJ, Steen VD, et al. The modified Rodnan skin score is an accurate reflection of skin biopsy thickness in systemic sclerosis. J Rheumatol. 1998; 25: 84-8.

7. Deepa AS, Rachel RP, Ramchandran P, et al. Pulmonary involvement in systemic sclero- 
sis: A clinical profile. Lung India. 2016; 33: 144-7.

8. D'Andrea A, Bellissimo S, Scotto di Uccio $\mathrm{F}$, et al. Associations of right ventricular myocardial function with skin and pulmonary involvement in asymptomatic patients with systemic sclerosis. Ital Heart J. 2004; 5: 831-9.

9. Medsger TA, Jr., Bombardieri S, Czirjak L, et al. Assessment of disease severity and prognosis. Clin Exp Rheumatol. 2003; 21: S42-6.

10. Torok KS, Baker NA, Lucas M, et al. Reliability and validity of the delta finger-to-palm (FTP), a new measure of finger range of motion in systemic sclerosis. Clin Exp Rheumatol. 2010; 28: S28-36.

11. van den Hoogen F, Khanna D, Fransen J, et al. 2013 classification criteria for systemic sclerosis: an American College of Rheumatology/ European League against Rheumatism collaborative initiative. Arthritis Rheum. 2013; 65: 2737-47.

12. Ghosh D. Incorporating the empirical null hypothesis into the Benjamini-Hochberg procedure. Stat Appl Genet Mol Biol. 2012; 11: 4.

13. Khanna PP, Furst DE, Clements PJ, et al. Tendon friction rubs in early diffuse systemic sclerosis: prevalence, characteristics and longitudinal changes in a randomized controlled trial. Rheumatology. 2010; 49: 955-9.

14. Dobrota R, Maurer B, Graf N, et al. Prediction of improvement in skin fibrosis in diffuse cutaneous systemic sclerosis: a EUSTAR analysis. Annals Rheum Dis. 2016; 75: 1743-8.

15. Arslan Tas D, Erken E, Sakalli H, Yucel AE. Evaluating hand in systemic sclerosis. Rheumatol Int. 2012; 32: 3581-6.
16. Doré A, Lucas M, Ivanco D, et al. Significance of palpable tendon friction rubs in early diffuse cutaneous systemic sclerosis. Arthritis Care Res. 2013; 65: 1385-9.

17. Avouac J, Walker U, Tyndall A, et al. Characteristics of joint involvement and relationships with systemic inflammation in systemic sclerosis: results from the EULAR Scleroderma Trial and Research Group (EUSTAR) database. J Rheumatol. 2010; 37: 1488-501.

18. Wangkaew S, Sivasomboon C, Leungwatthananon W, et al. Prevalence and predictors of hand involvement in Thai patients with systemic sclerosis. Int J Rheum Dis. 2018; 21: 240-8.

19. Csiki Z, Garai I, Varga J, et al. Microcirculation of the fingers in Raynaud's syndrome: (99m)Tc-DTPA imaging. Nuklearmedizin. 2005; 44: 29-32.

20. Galuska L, Garai I, Csiki Z, et al. The clinical usefulness of the fingers-to-palm ratio in different hand microcirculatory abnormalities. Nucl Med Commun. 2000; 21: 659-63.

21. Wakker J, Kratzer W, Graeter T, Schmidberger J. Elasticity standard values of the Achilles tendon assessed with acoustic radiation force impulse elastography on healthy volunteers: a cross section study. BMC Musculoskelet Disord. 2018; 19: 139.

22. Budulgan M, Dilek B, Dag SB, et al. Relationship between serum leptin level and disease activity in patients with systemic sclerosis. Clin Rheumatol. 2014; 33: 335-9.

23. Ross L, Stevens W, Rabusa C, et al. The role of inflammatory markers in assessment of disease activity in systemic sclerosis. Clin Exp Rheumatol. 2018; 36: 126-34. 